\title{
B.-Y. CHEN INEQUALITIES FOR SEMISLANT SUBMANIFOLDS IN SASAKIAN SPACE FORMS
}

\author{
DRAGOS CIOROBOIU
}

Received 20 December 2001

\begin{abstract}
Chen (1993) established a sharp inequality for the sectional curvature of a submanifold in Riemannian space forms in terms of the scalar curvature and squared mean curvature. The notion of a semislant submanifold of a Sasakian manifold was introduced by J. L. Cabrerizo, A. Carriazo, L. M. Fernandez, and M. Fernandez (1999). In the present paper, we establish Chen inequalities for semislant submanifolds in Sasakian space forms by using subspaces orthogonal to the Reeb vector field $\xi$.
\end{abstract}

2000 Mathematics Subject Classification: 53C40, 53B25, 53D15.

1. Preliminaries: Riemannian invariants. The Riemannian invariants of a Riemannian manifold are the intrinsic characteristics of the Riemannian manifold. In this section, we recall a string of Riemannian invariants on a Riemannian manifold [5].

Let $M$ be a Riemannian manifold. Denote by $K(\pi)$ the sectional curvature of $M$ associated with a plane section $\pi \subset T_{p} M, p \in M$.

For any orthonormal basis $\left\{e_{1}, e_{2}, \ldots, e_{n}\right\}$ of the tangent space $T_{p} M$, the scalar curvature $\tau$ at $p$ is defined by

$$
\tau(p)=\sum_{i<j} K\left(e_{i} \wedge e_{j}\right)
$$

One denotes

$$
(\inf K)(p)=\inf \left\{K(\pi) \mid \pi \subset T_{p} M, \operatorname{dim} \pi=2\right\}
$$

and introduces the Chen invariant

$$
\delta_{M}(p)=\tau(p)-(\inf K)(p) .
$$

We recall the following lemma of Chen [4]. 
LEMMA 1.1. Let $a_{1}, \ldots, a_{n}, c$ be $n+1$ real numbers, where $n \geq 2$, such that

$$
\left(\sum_{i=1}^{n} a_{i}\right)^{2}=(n-1)\left(\sum_{i=1}^{n} a_{i}^{2}+c\right)
$$

Then $2 a_{1} a_{2} \geq c$ and the equality holds if and only if $a_{1}+a_{2}=a_{3}=\cdots=a_{n}$.

Let $M$ be an $n$-dimensional submanifold of a Riemannian manifold $\tilde{M}$. We denote by $\nabla$ the Riemannian connection of $M$. Also, let $h$ be the second fundamental form of $M$ and let $R$ (resp., $\tilde{R}$ ) be the Riemann curvature tensor of $M$ (resp., $\tilde{M}$ ).

Then the equation of Gauss is given by

$$
\begin{aligned}
\tilde{R}(X, Y, Z, W)= & R(X, Y, Z, W)+g(h(X, W), h(Y, Z)) \\
& -g(h(X, Z), h(Y, W)),
\end{aligned}
$$

for any vectors $X, Y, Z$, and $W$ tangent to $M$, where we denote as usual $R(X, Y$, $Z, W)=-g(R(X, Y) Z, W)$.

Let $p \in M$ and $\left\{e_{1}, \ldots, e_{n}\right\}$ an orthonormal basis of the tangent space $T_{p} M$. We denote by $H$ the mean curvature vector, that is,

$$
H(p)=\frac{1}{n} \sum_{i=1}^{n} h\left(e_{i}, e_{i}\right)
$$

Also, we set

$$
h_{i j}^{r}=g\left(h\left(e_{i}, e_{j}\right), e_{r}\right), \quad\|h\|^{2}=\sum_{i, j=1}^{n} g\left(h\left(e_{i}, e_{j}\right), h\left(e_{i}, e_{j}\right)\right) .
$$

2. Semislant submanifolds in Sasakian manifolds. A $(2 m+1)$-dimensional Riemannian manifold $(\tilde{M}, g)$ is said to be a Sasakian manifold if it admits an endomorphism $\phi$ of its tangent bundle $T \tilde{M}$, a vector field $\xi$, and a 1 -form $\eta$ satisfying (see, e.g., [7])

$$
\begin{gathered}
\phi^{2}=-I d+\eta \otimes \xi, \quad \eta(\xi)=1, \quad \phi \xi=0, \quad \eta \circ \phi=0, \\
g(\phi X, \phi Y)=g(X, Y)-\eta(X) \eta(Y), \quad \eta(X)=g(X, \xi), \\
\left(\tilde{\nabla}_{X} \phi\right) Y=g(X, Y) \xi-\eta(Y) X, \quad \tilde{\nabla}_{X} \xi=-\phi X,
\end{gathered}
$$

for any vector fields $X$ and $Y$ on $T \tilde{M}$, where $\tilde{\nabla}$ denotes the Riemannian connection with respect to $g$.

A plane section $\pi$ in $T_{p} \tilde{M}$ is called a $\phi$-section if it is spanned by $X$ and $\phi X$, where $X$ is a unit tangent vector orthogonal to $\xi$. The sectional curvature of a $\phi$-section is called a $\phi$-sectional curvature. A Sasakian manifold with constant $\phi$-sectional curvature $c$ is said to be a Sasakian space form and is denoted by $\tilde{M}(c)$. 
The curvature tensor of $\tilde{M}(c)$ of a Sasakian space form $\tilde{M}(c)$ is given by (see [1])

$$
\begin{aligned}
\tilde{R}(X, Y) Z= & \frac{c+3}{4}\{g(Y, Z) X-g(X, Z) Y\} \\
+ & \frac{c-1}{4}\{\eta(X) \eta(Z) Y-\eta(Y) \eta(Z) X+g(X, Z) \eta(Y) \xi-g(Y, Z) \eta(X) \xi \\
& +g(\phi Y, Z) \phi X-g(\phi X, Z) \phi Y-2 g(\phi X, Y) \phi Z\},
\end{aligned}
$$

for any tangent vector fields $X, Y$, and $Z$ on $\tilde{M}(c)$.

As examples of Sasakian space forms, we mention $\mathbb{R}^{2 m+1}$ and $S^{2 m+1}$, with standard Sasakian structures (see [1]).

DEFINITION 2.1 [2]. A differentiable distribution $\mathscr{D}$ on $M$ is called a slant distribution if for each $x \in M$ and each nonzero vector $X \in \mathscr{D}_{x}$, the angle $\theta_{\mathscr{D}}(X)$ between $\phi X$ and the vector subspace $\mathscr{D}_{X}$ is constant, which is independent of the choice of $x \in M$ and $X \in \mathscr{D}_{x}$. In this case, the constant angle $\theta_{\mathscr{D}}$ is called the slant angle of the distribution $\mathscr{D}$.

DEFINITION 2.2 [2]. A submanifold $M$ tangent to $\xi$ is said to be a bislant submanifold of $\tilde{M}$ if there exist two orthogonal distributions $\mathscr{D}_{1}$ and $\mathscr{D}_{2}$ on $M$ such that

(i) $T M$ admits the orthogonal direct decomposition $T M=\mathscr{D}_{1} \oplus \mathscr{D}_{2} \oplus\{\xi\}$;

(ii) for any $i=1,2, \mathscr{D}_{i}$ is slant distribution with slant angle $\theta_{i}$.

DEFINITION 2.3 [2]. A submanifold $M$ tangent to $\xi$ is said to be a semislant submanifold of $\tilde{M}$ if there exist two orthogonal distributions $\mathscr{D}_{1}$ and $\mathscr{D}_{2}$ on $M$ such that

(i) $T M$ admits the orthogonal direct decomposition $T M=\mathscr{D}_{1} \oplus \mathscr{D}_{2} \oplus\{\xi\}$;

(ii) the distribution $\mathscr{D}_{1}$ is an invariant distribution, that is, $\phi\left(\mathscr{D}_{1}\right)=\mathscr{D}_{1}$;

(iii) the distribution $\mathscr{D}_{2}$ is slant with angle $\theta \neq 0$.

DEFinITION 2.4 [3]. A submanifold $M$ is said to be a slant if for any $x \in M$ and any $X \in T_{x} M$, linearly independent on $\xi$, the angle between $\phi X$ and $T_{x} M$ is a constant $\theta \in[0, \pi / 2]$, called the slant angle of $M$ in $\tilde{M}$.

Invariant and anti-invariant immersions are slant immersions with slant angles $\theta=0$ and $\theta=\pi / 2$, respectively. A slant immersion which is neither invariant nor anti-invariant is called a proper slant immersion (see [3]).

In [2], the invariant distribution of a semislant submanifold is a slant distribution with zero angle. Thus, it is obvious that, in fact, semislant submanifolds are particular cases of bislant submanifolds. Moreover, it is clear that if $\theta=\pi / 2$, then the semislant submanifold is a semi-invariant submanifold.

(a) If $\mathscr{D}_{2}=0$, then $M$ is an invariant submanifold.

(b) If $\mathscr{D}_{1}=0$ and $\theta=\pi / 2$, then $M$ is an anti-invariant submanifold. 
(c) If $\mathscr{D}_{1}=0$ and $\theta \neq \pi / 2$, then $M$ is a proper slant submanifold with slant angle $\theta$.

A semislant submanifold is said to be proper if both $\mathscr{D}_{1}$ and $\mathscr{D}_{2}$ are nontrivial and $\theta \neq \pi / 2$.

For the properties and examples of semislant submanifolds in Sasakian manifolds, we refer to [2].

For any tangent vector field $X$ to $M$, one decomposes $\phi X=P X+F X$, where $P X$ and $F X$ are the tangential and normal components of $\phi X$, respectively. We denote

$$
\|P\|^{2}=\sum_{i, j=1}^{n} g^{2}\left(P e_{i}, e_{j}\right) .
$$

3. B.-Y. Chen inequality. We prove a Chen inequality for proper semislant submanifolds in a Sasakian space form. We consider plane sections $\pi$ orthogonal to $\xi$ and invariant by $P$. We denote $\operatorname{dim} \mathscr{D}_{1}=2 d_{1}$ and $\operatorname{dim} \mathscr{D}_{2}=2 d_{2}$.

THEOREM 3.1. Let $M$ be an n-dimensional proper semislant submanifold in $a(2 m+1)$-dimensional Sasakian space form $\tilde{M}(c)$. Then,

$$
\begin{aligned}
K(\pi) \geq & \tau-\frac{n-2}{2}\left\{\frac{n^{2}}{n-1}\|H\|^{2}+\frac{(c+3)(n+1)}{4}\right\} \\
& -\frac{(c-1)}{4}\left[3 d_{2} \cos ^{2} \theta+3\left(d_{1}-1\right)-(n-1)\right],
\end{aligned}
$$

for any plane section $\pi$ invariant by $P$ and tangent to $\mathscr{D}_{1}$, and

$$
\begin{aligned}
K(\pi) \geq & \tau-\frac{n-2}{2}\left\{\frac{n^{2}}{n-1}\|H\|^{2}+\frac{(c+3)(n+1)}{4}\right\} \\
& -\frac{(c-1)}{4}\left[3\left(d_{2}-1\right) \cos ^{2} \theta+3 d_{1}-(n-1)\right],
\end{aligned}
$$

for any plane section $\pi$ invariant by $P$ and tangent to $\mathscr{D}_{2}$.

The equality case of inequalities (3.1) and (3.2) holds at a point $p \in M$ if and only if there exists an orthonormal basis $\left\{e_{1}, \ldots, e_{n}=\xi\right\}$ of $T_{p} M$ and an orthonormal basis $\left\{e_{n+1}, \ldots, e_{2 m}, e_{2 m+1}\right\}$ of $T_{p}^{\perp} M$ such that the shape operators of $M$ in $\tilde{M}(c)$ at $p$ have the following forms:

$$
\begin{aligned}
A_{n+1} & =\left(\begin{array}{cccccccc}
a & 0 & 0 & \cdot & \cdot & \cdot & 0 \\
0 & b & 0 & \cdot & \cdot & \cdot & 0 \\
0 & 0 & & & \mu I_{n-2} & &
\end{array}\right), \quad a+b=\mu, \\
A_{r} & =\left(\begin{array}{ccccccc}
h_{11}^{r} & h_{12}^{r} & 0 & \cdot & \cdot & \cdot & 0 \\
h_{12}^{r} & -h_{11}^{r} & 0 & \cdot & \cdot & \cdot & 0 \\
0 & 0 & & & 0 & 0
\end{array}\right), \quad r \in\{n+2, \ldots, 2 m+1\} .
\end{aligned}
$$


Proof. We recall the Gauss equation for the submanifold $M$

$$
\begin{aligned}
\tilde{R}(X, Y, Z, W)= & R(X, Y, Z, W)+g(h(X, W), h(Y, Z)) \\
& -g(h(X, Z), h(Y, W)),
\end{aligned}
$$

for all $X, Y, Z, W \in \Gamma(T M)$.

Since $\tilde{M}(c)$ is a Sasakian space form, we have

$$
\begin{aligned}
\tilde{R}(X, Y, Z, W)=\frac{c+3}{4}\{- & g(Y, Z) g(X, W)+g(X, Z) g(Y, W)\} \\
+\frac{c-1}{4}\{ & -\eta(X) \eta(Z) g(Y, W)+\eta(Y) \eta(Z) g(X, W) \\
& -g(X, Z) \eta(Y) g(\xi, W)+g(Y, Z) \eta(X) g(\xi, W) \\
& -g(\phi Y, Z) g(\phi X, W)+g(\phi X, Z) g(\phi Y, W) \\
& +2 g(\phi X, Y) g(\phi Z, W)\}, \quad \forall X, Y, Z, W \in \Gamma(T M) .
\end{aligned}
$$

Let $p \in M,\left\{e_{1}, \ldots, e_{n}=\xi\right\}$ an orthonormal basis of $T_{p} M$, and $\left\{e_{n+1}, \ldots\right.$, $\left.e_{2 m}, e_{2 m+1}\right\}$ an orthonormal basis of $T_{p}^{\perp} M$. For $X=Z=e_{i}$ and $Y=W=e_{j}$, $\forall i, j \in\{1, \ldots, n\}$, from (3.5), it follows that

$$
\begin{aligned}
\sum_{i, j} \tilde{R}\left(e_{i}, e_{j}, e_{i}, e_{j}\right)= & \frac{c+3}{4}\left(-n+n^{2}\right) \\
& +\frac{c-1}{4}\left\{-2(n-1)+3 \sum_{i, j=1}^{n} g^{2}\left(\phi e_{i}, e_{j}\right)\right\} .
\end{aligned}
$$

Let $M$ be a proper semislant submanifold of $\tilde{M}(c)$ and $\operatorname{dim} M=n=2 d_{1}+$ $2 d_{2}+1$. We consider an adapted semislant orthonormal frames

$$
\begin{aligned}
e_{1}, e_{2} & =\phi e_{1}, \ldots, e_{2 d_{1}-1}, e_{2 d_{1}}=\phi e_{2 d_{1}-1} \\
e_{2 d_{1}+1}, e_{2 d_{1}+2} & =\frac{1}{\cos \theta} P e_{2 d_{1}+1}, \ldots, e_{2 d_{1}+2 d_{2}-1}, e_{2 d_{1}+2 d_{2}} \\
& =\frac{1}{\cos \theta} P e_{2 d_{1}+2 d_{2}-1}, e_{2 d_{1}+2 d_{2}+1} \\
& =\xi .
\end{aligned}
$$

Obviously, we have

$$
g^{2}\left(\phi e_{i}, e_{i+1}\right)= \begin{cases}1, & \text { for } i \in\left\{1, \ldots, 2 d_{1}-1\right\}, \\ \cos ^{2} \theta, & \text { for } i \in\left\{2 d_{1}+1, \ldots, 2 d_{1}+2 d_{2}-1\right\} .\end{cases}
$$

Then

$$
\sum_{i, j=1}^{n} g^{2}\left(\phi e_{i}, e_{j}\right)=2\left(d_{1}+d_{2} \cos ^{2} \theta\right)
$$


Relation (3.6) implies that

$$
\sum_{i, j} \tilde{R}\left(e_{i}, e_{j}, e_{i}, e_{j}\right)=\frac{c+3}{4}\left(n^{2}-n\right)+\frac{c-1}{4}\left[6\left(d_{1}+d_{2} \cos ^{2} \theta\right)-2(n-1)\right] .
$$

Denoting

$$
\|h\|^{2}=\sum_{i, j=1}^{n} g\left(h\left(e_{i}, e_{j}\right), h\left(e_{i}, e_{j}\right)\right)
$$

from relation (3.10), one has

$$
\frac{c+3}{4} n(n-1)+\frac{c-1}{4}\left[6\left(d_{1}+d_{2} \cos ^{2} \theta\right)-2 n+2\right]=2 \tau-n^{2}\|H\|^{2}+\|h\|^{2}
$$

or, equivalently,

$$
2 \boldsymbol{\tau}=n^{2}\|H\|^{2}-\|h\|^{2}+\frac{c+3}{4} n(n-1)+\frac{c-1}{4}\left[6\left(d_{1}+d_{2} \cos ^{2} \theta\right)-2 n+2\right] .
$$

If we put

$$
\begin{aligned}
\varepsilon= & 2 \tau-\frac{n^{2}}{n-1}(n-2)\|H\|^{2} \\
& -\frac{c+3}{4} n(n-1)-\frac{c-1}{4}\left[6\left(d_{1}+d_{2} \cos ^{2} \theta\right)-2 n+2\right],
\end{aligned}
$$

we obtain

$$
n^{2}\|H\|^{2}=(n-1)\left(\varepsilon+\|h\|^{2}\right)
$$

Let $p \in M, \pi \subset T_{p} M, \operatorname{dim} \pi=2$, and $\pi$ orthogonal to $\xi$ and invariant by $P$. We consider two cases.

(i) The plane section $\pi$ is tangent to $\mathscr{D}_{1}$. We may assume that $\pi=\operatorname{sp}\left\{e_{1}, e_{2}\right\}$. We choose $e_{n+1}=H /\|H\|$. Relation (3.15) becomes

$$
\left(\sum_{i=1}^{n} h_{i i}^{n+1}\right)^{2}=(n-1)\left\{\sum_{i, j=1}^{n} \sum_{r=n+1}^{2 m+1}\left(h_{i j}^{r}\right)^{2}+\varepsilon\right\}
$$

or, equivalently,

$$
\left(\sum_{i=1}^{n} h_{i i}^{n+1}\right)^{2}=(n-1)\left\{\sum_{i=1}^{n}\left(h_{i i}^{n+1}\right)^{2}+\sum_{i \neq j}\left(h_{i j}^{n+1}\right)^{2}+\sum_{r=n+2}^{2 m+1} \sum_{i, j=1}^{n}\left(h_{i j}^{r}\right)^{2}+\varepsilon\right\} .
$$


Using Lemma 1.1, we derive from (3.17)

$$
2 h_{11}^{n+1} h_{22}^{n+1} \geq \sum_{i \neq j}\left(h_{i j}^{n+1}\right)^{2}+\sum_{i, j=1}^{n} \sum_{r=n+2}^{2 m+1}\left(h_{i j}^{r}\right)^{2}+\varepsilon .
$$

From the Gauss equation for $X=Z=e_{1}$ and $Y=W=e_{2}$, we obtain

$$
\begin{aligned}
K(\pi)= & \frac{c+3}{4}+3 \cdot \frac{c-1}{4}+\sum_{r=n+1}^{2 m+1}\left[h_{11}^{r} h_{22}^{r}-\left(h_{12}^{r}\right)^{2}\right] \\
\geq & \frac{c+3}{4}+3 \cdot \frac{c-1}{4}+\frac{1}{2} \sum_{i \neq j}\left(h_{i j}^{n+1}\right)^{2}+\frac{1}{2} \sum_{i, j=1}^{n} \sum_{r=n+2}^{2 m+1}\left(h_{i j}^{r}\right)^{2} \\
& +\frac{\varepsilon}{2}+\sum_{r=n+2}^{2 m+1} h_{11}^{r} h_{22}^{r}-\sum_{r=n+1}^{2 m+1}\left(h_{12}^{r}\right)^{2} \\
= & \frac{c+3}{4}+3 \cdot \frac{c-1}{4}+\frac{1}{2} \sum_{i \neq j}\left(h_{i j}^{n+1}\right)^{2}+\frac{1}{2} \sum_{r=n+2}^{2 m+1} \sum_{i, j>2}\left(h_{i j}^{r}\right)^{2} \\
& +\frac{1}{2} \sum_{r=n+2}^{2 m+1}\left(h_{11}^{r}+h_{22}^{r}\right)^{2}+\sum_{j>2}\left[\left(h_{1 j}^{n+1}\right)^{2}+\left(h_{2 j}^{n+1}\right)^{2}\right]+\frac{\varepsilon}{2} \\
\geq & \frac{c+3}{4}+3 \cdot \frac{c-1}{4}+\frac{\varepsilon}{2}
\end{aligned}
$$

or, equivalently,

$$
K(\pi) \geq \frac{c+3}{4}+3 \cdot \frac{c-1}{4}+\frac{\varepsilon}{2} .
$$

If we substitute (3.14) into (3.20), we obtain (3.1).

(ii) Similarly, if $\pi$ is tangent to $\mathscr{D}_{2}$, we obtain (3.2).

The case of equality at a point $p \in M$ holds if and only if it achieves the equalities in inequalities (3.18), (3.19), and (3.20) and in Lemma 1.1. Then

$$
\begin{aligned}
& h_{i j}^{n+1}=0, \quad \forall i \neq j, i, j>2, \\
& h_{i j}^{r}=0, \quad \forall i \neq j, i, j>2, r=n+1, \ldots, 2 m+1, \\
& h_{11}^{r}+h_{22}^{r}=0, \quad \forall r=n+2, \ldots, 2 m+1, \\
& h_{1 j}^{n+1}=h_{2 j}^{n+1}=0, \quad \forall j>2, \\
& h_{11}^{n+1}+h_{22}^{n+1}=h_{33}^{n+1}=\cdots=h_{n n}^{n+1} .
\end{aligned}
$$

We may choose $\left\{e_{1}, e_{2}\right\}$ such that $h_{12}^{n+1}=0$ and we denote $a=h_{11}^{r}, b=h_{22}^{r}$, and $\mu=h_{33}^{n+1}=\cdots=h_{n n}^{n+1}$.

It follows that the shape operators take the desired forms.

Chen inequality for slant submanifolds in Sasakian space forms was proved in [6]. 
THEOREM 3.2 [6]. Let $M$ be an n-dimensional $\theta$-slant submanifold in a ( $2 m+$ 1)-dimensional Sasakian space form $\tilde{M}(c)$. Then,

$$
\begin{aligned}
\delta_{M} \leq & \frac{n-2}{2}\left\{\frac{n^{2}}{n-1}\|H\|^{2}+\frac{(c+3)(n+1)}{4}\right\} \\
& +\frac{(c-1)}{8}\left[3(n-3) \cos ^{2} \theta-2(n-1)\right] .
\end{aligned}
$$

The equality case of inequality (3.1) holds at a point $p \in M$ if and only if there exists an orthonormal basis $\left\{e_{1}, \ldots, e_{n}=\xi\right\}$ of $T_{p} M$ and an orthonormal basis $\left\{e_{n+1}, \ldots, e_{2 m}, e_{2 m+1}\right\}$ of $T_{p}^{\perp} M$ such that the shape operators of $M$ in $\tilde{M}(c)$ at $p$ have the following forms:

$$
\begin{aligned}
A_{n+1} & =\left(\begin{array}{cccccccc}
a & 0 & 0 & \cdot & \cdot & \cdot & 0 \\
0 & b & 0 & \cdot & \cdot & \cdot & 0 \\
0 & 0 & & & \mu I_{n-2} & &
\end{array}\right), \quad a+b=\mu, \\
A_{r} & =\left(\begin{array}{ccccccc}
h_{11}^{r} & h_{12}^{r} & 0 & \cdot & \cdot & \cdot & 0 \\
h_{12}^{r} & -h_{11}^{r} & 0 & \cdot & . & \cdot & 0 \\
0 & 0 & & & 0 & 0
\end{array}\right), \quad r \in\{n+2, \ldots, 2 m+1\} .
\end{aligned}
$$

\section{REFERENCES}

[1] D. E. Blair, Contact Manifolds in Riemannian Geometry, Lecture Notes in Mathematics, vol. 509, Springer-Verlag, Berlin, 1976.

[2] J. L. Cabrerizo, A. Carriazo, L. M. Fernández, and M. Fernández, Semi-slant submanifolds of a Sasakian manifold, Geom. Dedicata 78 (1999), no. 2, 183-199.

[3] _ Slant submanifolds in Sasakian manifolds, Glasgow Math. J. 42 (2000), no. $1,125-138$.

[4] B.-Y. Chen, Some pinching and classification theorems for minimal submanifolds, Arch. Math. (Basel) 60 (1993), no. 6, 568-578.

[5] _ Some new obstructions to minimal and Lagrangian isometric immersions, Japan. J. Math. (N.S.) 26 (2000), no. 1, 105-127.

[6] D. Cioroboiu and A. Oiaga, B. Y. Chen inequalities for slant submanifolds in Sasakian space forms, to appear in Rend. Circ. Mat. Palermo 51, 2002.

[7] K. Yano and M. Kon, Structures on Manifolds, Series in Pure Mathematics, vol. 3, World Scientific Publishing, Singapore, 1984.

Dragoş Cioroboiu: Department of Mathematics I, University of Politehnica of Bucharest, Splaiul Independenței 313, 77206 Bucharest, Romania

E-mail address: ti abaprov@pcnet.ro 


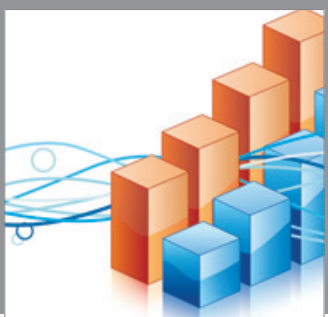

Advances in

Operations Research

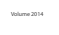

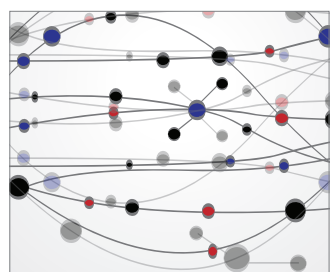

\section{The Scientific} World Journal
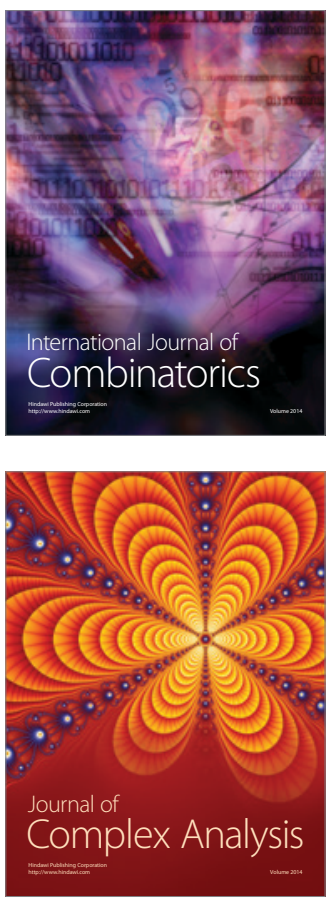

International Journal of

Mathematics and

Mathematical

Sciences
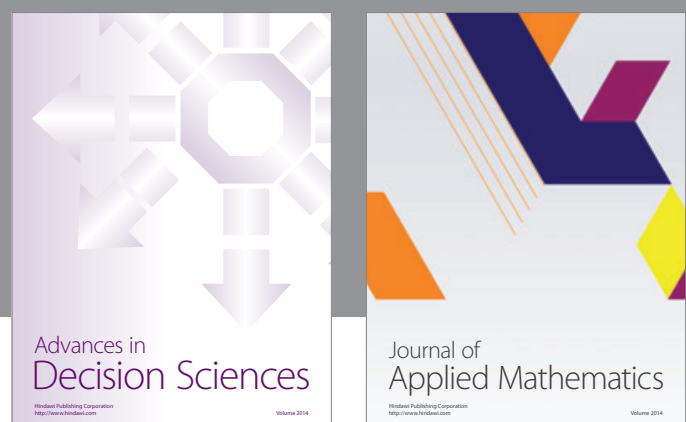

Journal of

Applied Mathematics
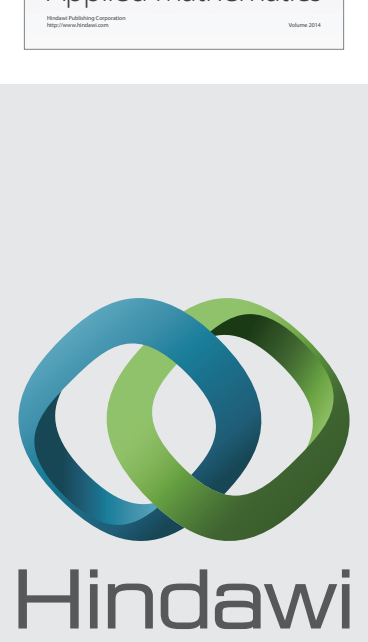

Submit your manuscripts at http://www.hindawi.com
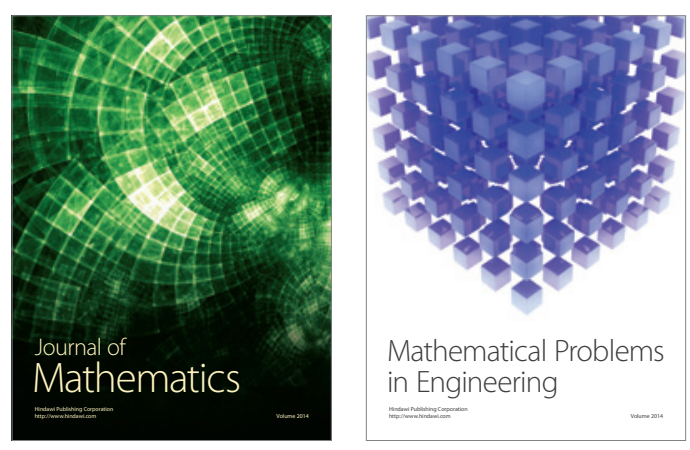

Mathematical Problems in Engineering
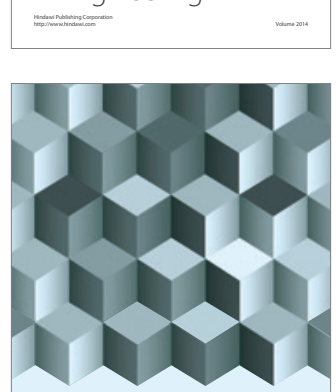

Journal of

Function Spaces
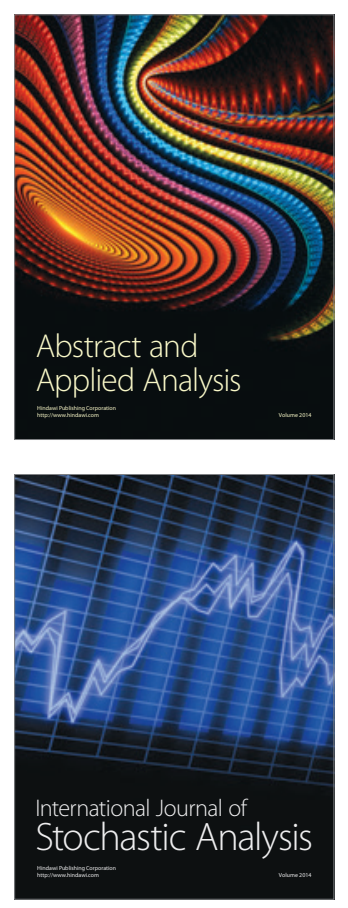

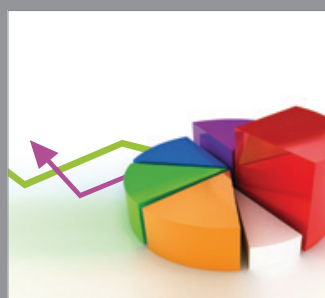

ournal of

Probability and Statistics

Promensencen
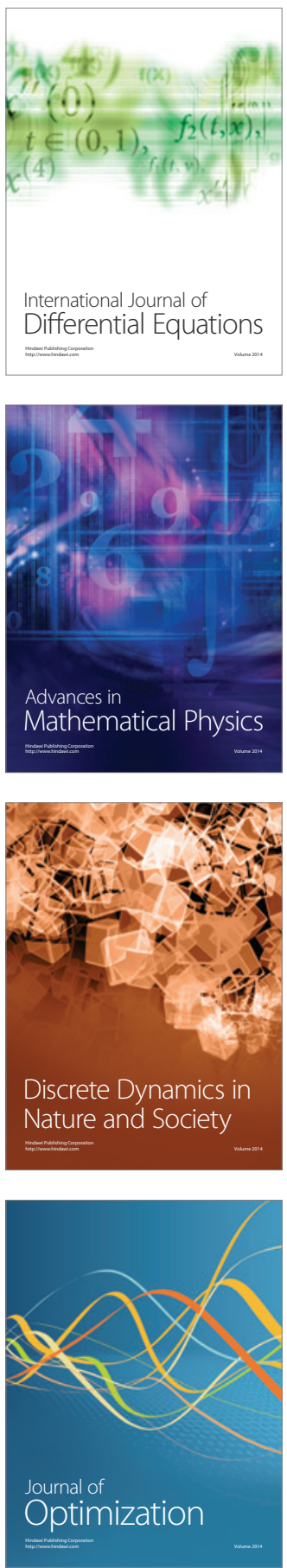